XII.

\title{
Ueber Kriegsneurosen, ihre Prognose und Behandlung.
}

\author{
Von \\ Prof. Dr. Rosenfeld, \\ Stabsarzt d. R. und Chefarzt des Fest.-Laz. 21 Strassburg i. Ils.
}

In einem Lazarett, welches vornehmlich die Aufgabe hat, Neurosen nach Kriegsbeschädigungen durch Heilbeschäftigung günstig zu beeinflussen und auf den Grad ihrer Dienstfähigkeit zu prüfen, findet sich naturgemäss ein Material von Fällen zusammen, bei denen meist nur die leichteren Grade abnormer psychischer und nervöser Reaktionen zu beobachten sind. Unser klinisches Interesse braucht dieser Gruppe von leichteren Fällen von Kriegsneurosen gegenüber nicht geringer zu sein, als den Fällen, in denen wir voll entwickelte Krankheitsbilder vor uns haben. Geben uns doch gerade diese leichteren Fälle Gelegenheit, die Anfangsstadien krankhafter nervöser Mechanismen genauer zu studieren, und die Möglichkeit, die verschiedenen Typen von nervösen Reaktionen leichter auseinander zu halten. Ferner ist die Zahl der Fälle, in denen sich durch Heilbeschäftigung volle oder teilweise Dienstfähigkeit erreichen lasst, recht beachtenswert, wie ich dies bereits in einer früheren Publikation$^{1}$ ) gezeigt habe. So haben diese Fälle auch ein praktisches Interesse.

Im Laufe eines Jahres sind im Lazarett 21 über 1100 Fälle zur Beobachtung und Behandlung gekommen. Die unten gewählte Gruppierung dieses gesamten Materials obne Auswahl der Fälle hat sich zwanglos aus den tatsächlich vorhandenen Symptomen und den am hä̈ufigsten vorkommenden Kombinationen derselben ergeben. Die Aufstellung verschiedener Gruppen, welche sich oft nur durch einzelne Symptome von einander unterscheiden, könnte auf den ersten Blick vielleicht befremden,

1) Vgl. Rosenfeld, Ueber die Erfolge der Heilbeschäftigung im Lazarett. Strassburger Med.-Ztg. 1916. H. 3. - Wollenberg, Ueber den Dienstbetrieb im Lazarett und die Einteilung derLente zur Arbeit. Deutsche med. Wochenschr. 1915. Nr. 26. 1916. Nr. 1. 
da man die nervösen Reaktionen nur auf einige wenige Grundtypen zurückzuführen pflegt. Ich verfolge aber mit der unten gewählten Einteilung des gesamten Krankenmaterials meines Lazaretts mehr praktischdiagnostische $Z$ wecke. Es soll gezeigt werden, in welchen Kombinationen die nervösen Reaktionen bei Kriegsbeschädigten am häufigsten vorzukommen pflegen, wie die Prognose mancher Fälle durch das Hinzutreten dieses oder jenes an sich unwesentlich erscheinenden Symptoms oder Umstandes bestimmt wird, und welche Fälle einer Heilung bis zur Dienstfähigkeit zugänglich erscheinen und welche nicht. Es ergeben sich aus dieser Darstellung gewisse Anhaltspunkte für die Entlassung und frühzeitige Erkennung auf Dienstuntauglichkeit.

\section{Gruppe 1: Einfache nervöse Erschöpfung.}

Als Ausgangspunkt meiner Zusammenstellung wähle ich die Fälle, in denen als eimzige Grundstörung eine abnorme Erregbarkeit und Erschöpfbarkeit körperlich-nervöser und psychischer Funktionen nachweisbar sind und alle weiteren nervösen Reaktionen fehlen. $\mathrm{Zu}$ diesen Symptomen gehören bekanntlich: Lebhafte Steigerung aller Haut- und Sehnenreflexe, leichtes Zittern der Hände and des Körpers, etwas gesteigerte Schweissabsonderung, feuchte kühle Hände, lebhafte seelische Erregbarkeit bei geringfügigen Anlässen, lebhafte, etwas hastige Ausdrucksbewegungen, leichte körperliche und seelische Ermüdbarkeit Schlafstörungen, leicht auslösbare Angstempfindungen, Schwindel empfindungen beim Bücken und bei körperlichen Anstrengungen.

Die Zahl solcher Fälle war beträchtlich. Unter 1123 fanden sich 411. Sie sind in ibrem Mechanismus uns insofern verständlich, als jedes der genannten Symptome infolge körperlicher Ueberanstrengung oder heftiger seelischer Erregung schliesslich bei jedem gesunden Menschen auftreten und für kurze Zeit bestehen bleiben kann. Das Pathologische liegt also in diesen Fällen in der erleichtertel Auslösung solcher Reaktionen und in dem Persistieren derselben.

Bei der Entstehung dieser Ieichteren Fälle von rein neurasthenischem Typus spielen starke Anstrengungen während längerer Zeit, Märsche, ferner starke seelische Aufregungen bei langdanernder Beschiessung die Hauptrolle. In manchen Fällen wird man mit Rücksicht auf eine etwas schwächliche Konstitution auch dann eine latente Disposition zu diesen nervösen Störungen annehmen können, wenn im übrigen die Anamnese eigentlich keine Anhaltspunkte dafür gibt. Die Prognose dieser Fälle pflegt stets eine gute zu sein, wenn es sich um jüngere Leute, etwa bis zum 35. Jahre handelt und nervöse, namentlich psychopathische Antezedentien fehlen. 
Die Behandlung dieser Fälle bestand in Verrichten von Feld- und Gartenarbeiten, zu welchen sich diese Leute infolge der bei ihnen noch ungeschwächten Lust zur Betätigung von selbst drängten.

Gruppe 2: Einfache nervöse Erschöpfung mit lokalisierten Organbeschwerden.

Diese Gruppe ist dadurch charakterisiert, dass zu den Symptomen der einfachen nervösen Erschöpfung nervöse Beschwerden in einzelnen Organgebieten treten, welche uun ihrerseits ganz im Vordergrunde des. Krankheitsbildes stehen. Es sind dies Fälle mit funktionellen Herzbeschwerden, namentlich Tachykardien, mit Magenbeschwerden und Störungen der Nahrungsaufnahme infolge grosser Empfindlichkeit des. Magens und schliesslich Fälle mit stärker hervortretenden Störungen in einzelnen Abschnitten des Nervensystems. In allen Fällen war selbstverständlich eine spezialärztliche interne Untersuchung vorausgegangen. Es kamen 224 derartiger Fälle zur Beobachtung. Am häufigsten waren die Fälle mit emotionellen Tachykardien und Sensationen in der Herzgegend; in anderen Fällen war die Pulsbeschleunigung fast dauernd vorhanden oder erfuhr bei den leichtesten Körperanstrengungen eine erhebliche Steigerang. Dazu kamen in einzelnen Fällen allgemeineHyperidrosis, Blutandrang nach dem Kopf, leichte Schlängelung der Temporalarterien obne Blutdrucksteigerung, pulsatorische Erschütterung. des Kopfes, einzelner Extremitäten und der Bauchaorta, Tachypnoe und psychogene Dyspnoe. In den Fällen mit nervösen Magenbeschwerden, Brechneigung nach dem Essen und Sensationen in der Magengegend, in denen die psychogene Entstehung sich nachweisen liess, war die Prognose gut, während in den Fällen, in denen die allgemeine nervöse Erschöpfung die ausschliessliche Grundlage der Störung war, weniger günstige Resultate sich erreichen liessen und sich oft Rückfälle mit starken Gewichtsabnahmen einstellten.

Bei der Entstehung dieser Fälle mit lokalisierten Organbeschwerden spielt die Veranlagung dazu wohl schon eine erheblichere Rolle, als in den Fällen der ersten Gruppe. Manche Kranken geben zu, dass sie. schon früher bei dieser oder jener Gelegenheit lokalisierte nervöse Beschwerden gehabt haben. Die Zahl dieser Fälle mit nachweisbarer. Disposition wäre wohl sicher noch grösser, wenn man immer eine. wirklich zuverlässige Anamnese bekäme. Es gehören zu dieser Gruppe die Fälle von mehr oder weniger latenter, angeborener Neurasthenie. mit lokalisierten Beschwerden. Warum bald dieses bald jenes Organsystem von den Beschwerden befallen wird, lässt sich nicht sagen. Vielleicht handelt es sich um eine abnorme Empfindlichkeit einzelner 
Abschnitte im vegetativen Nervensystem, welche auf eine allgemeine Schädigung zuerst ansprechen und die verschiedenen Organbeschwerden vermitteln.

Die Prognose dieser Gruppe ist schon wesentlich schlechter, als die der Gruppe I, nawentlich wenn die Leute schon früher bestimmte nervöse Beschwerden gehabt hatten. Die Mehrzahl der Fälle kann nur mit leichteren Arbeiten in der Handarbeitsstube oder Handwerksstube beschäftigt werden. Es ist wünschenswert, dass solche Fälle möglichst lange von einer Lazarettbehandlung ferngehalten werden, dass sie in Revierbehandlung bleiben und möglichst bald unter Berücksichtigung ihrer Eigenart einen für sie passenden Dienst angewiesen erhalten.

Gruppe 3: Nervöse Erschöpfung mit depressiver Verstimmung.

$\mathrm{Zu}$ den Symptomen der nervösen Erschöpfung gesellen sich in manchen Fällen Zustände von ausgesprochener depressiver Verstimmung, Energielosigkeit, Unentschlossenheit, Eigenbeziehungen und Kleinheitsideen. Nur in 2 derartigen Fällen liess sich zeigen, dass schon früher zyklothyme oder manisch-depressive Phasen bestanden hatten. In der Mehrzahl der Fälle - 79 - hatte sich mit zunehmender nervöser Erschöpfung auch die Depression eingestellt, die sich dann im weiteren Verlauf infolge irgendwelcher äusseren Umstände, Schwierigkeiten im Dienst, Versagen im Dienst steigerte und sich so zum Teil als reaktive Depression charakterisierte. In Fällen ohne psychopathische Antezedentien war ein längerer Aufenthalt im Lazarett, Arbeitstherapie und Beurlaubung: in die Heimat von gutem Erfolg. Die Fälle, in welchen bypochondrische Vorstellungen überwogen, gaben eine ungünstige Prognose.

Gruppe 4: Nervöse Erschöpfung bei angeborener Psychopathie und geistiger Minderwertigkeit.

In dieser Gruppe fasse ich die Fälle (im ganzen 82) zusammen, bei denen neben den oft schon in der Ausbildungszeit bervortretenden neurasthenischen Beschwerden und Anzeichen abnormer nervöser Erschöpfbarkeit noch ausgesprochene Züge von Psychopathie und psychischer Minderwertigkeit bestehen. Hier sind zu nennen die Fälle mit konstitutioneller Verstimmung, mit Zwangsvorstellungen, abnormer Reizbarkeit und Emotivität, ferner Leute mit querulatorischer Veranlagung, sehr schlaffe verweichlichte Naturen und leicht Schwachsinnige mit geringer geistiger Regsamkeit und indolentem trägem Benehmell.

Derartige Fälle sind am zweckmässigsten möglichst bald als dienstunbrauchbar zu entlassen, da brauchbare Leistungen nicht zu erwarten sind. Viele dieser Kranken lassen jeden guten Willen vermissen und 
reizen noch durch Simulationsversuche und Uebertreibung zu einem robusten Vorgehen von seiten des Arztes und zur Anwendung von $\mathrm{Z}$ wang. Es sind aber wohl kaum in diesen Fällen disziplinäre oder therapeutische Erfolge zu erwarten. So fallen derartige Elemente nur dem Lazarett oder der Truppe zur Last und machen später selbst nach kurzer Diehstzeit Dienstbeschädigungsansprüche. Manche stellen direkt eine Gefahr für die Truppe dar.

Gruppe 5: Nervöse Erschöpfung mit motorischen Reizerscheinungen (mit allgemeinem oder aufeinzelne Extremitäten beschränktem Tremor und tikartigen Bewegungen).

Auch für diese Grappe gibt erst die allgemeine nervöse Erschöpfbarkeit und Erschöpfung die Grundlage zum Auftreten der Störungen ab. Die emotionelle resp. psychogene Entstehung der Störung ist in der überwiegenden Mehrzabl der Fälle mit Sicherheit anzunehmen. Schwere seelische Erschütterung nach Explosionen, starke Aufregungen mit Ueberanstrengung und Verschüttung sind die gewöhnlichsten Gelegenheitsursachen. Die Fälle, welche man als sog. Schreckneurosen bezeichnet hat, gehören hierher. Bei disponierten Lenten, d.h. bei a $b$. norm erregbaren und erscböpfbaren geben schon die geringfügigsten Ereignisse Veraulassung. zum Auftreten solcher motorischer Störungen. So sah ich z.B. bei einem Rekruten, welcher früher nie nervöse Störungen geboten hatte, aber eine sehr schwächliche Konstitution zeigte, nie anstrengende Arbeiten verrichtet hatte und am ersten Tage. seiner Einstellung zur Beobachtung auf seine Dienstfähigkeit eingewiesen war, nach einem einstündigen Versuch, in der Schreinerwerkstätte zu arbeiten, einen heftigen funktionellen Schütteltremor auftreten, welcher erst nach eịnigen Wochen Bettruhe wieder geschwunden war. Das aus der Unfallpraxis zur Genüge bekannte Missverhältnis zwischen der Schwere eines Traumas und der Intensität der darauf folgenden nervösen Störungen tritt uns bei dieser Gruppe von Kriegsbeschädigten ganz besonders häufig entgegen. $Z u$ den Zitterbewegungen in einzelnen Extremitäten, die sich häufig mit tikartigen Kontraktionen in einzelnen Muskelgruppen der Hals- und Rumpfmuskulatur kombinieren, tritt oft noch eine stammelnde, stockende Sprache mit nervöser Dyspnoe.

Die Zahl dieser Fälle war recht beträchtlich (53).

Entsprechend der Entstehung dieser motorischen Störung als Reaktion auf Schreck, starke seelische Erregung etc. hat jede Form der Psychotherapie hier ihre besten Resultate zu verzeichnen. Welche Methode angewendet werden soll, hängt wohl von der Individualität des Falles ab. Im allgemeinen hat sich aber die Isolierung dieser Fälle, völlige Bett- 
ruhe mit kräftigen Dosen von Beruligungsmitteln und tägliche hypnotische Sitzungen oder Wacbsuggestionen sehr bewährt und fast immer zum Ziel geführt, d.h. zum raschen Nachlassen und völligem Schwinden der motorischen Reizerscheinungen. Allerdings müssen die psychotherapeutischen Massuahmen mit grosser Konsequenz wochenlang durchgeführt werden. Dass es für die therapeutischen Erfolge von Wichtigkeit ist, dass der Kranke einen guten Willen zeigt, selbst den Wunsch hat, gesund zu werden, und Vertrauen zu der Wirksamkeit der Methode hat, ist zur Genüge bekannt. So habe ich die besten psychotherapeutischen Erfolge bis zur Kriegsverwendungsfähigkeit bei aktiven Offizieren gehabt. Nach dem Schwinden der motorischen Reizerscheinungen ist es wichtig, dass die Rekonvaleszenten einen geeigneten Dienst zugewiesen erbalten und nicht sofort wieder in aufregende Situationen kommen, da sonst zunäcbst Rückfälle zu befürchten sind.

Gruppe 6: Fälle mit Bewusstseinstrübungen und Krampfzuständen.

Unter diesen Leuten, bei denen infolge oder während des Kriegsdienstes Krampfanfälle und Zustände von Bewusstseinstrübungen aufgetreten waren - im ganzen 193 Fälle mit Ausschluss der Epileptiker konnten folgende Gruppen unterschieden werden:

a) Hysterische Dämmerzustände und Anfälle.

b) Affektive Dämmerzustände.

c) Schwindelzustände mit leichten Bewusstseinsverlusten (Ohnmachten) obne Krampfanfälle bei allgemeiner nervöser Erschöpfung.

d) Anfälle von Bewusstlosigkeit bei beginnenden Veränderungen am Gefässsystem.

e) Anfälle von Bewusstlosigkeit und Krämpfen bei beginnender Hirnlues.

Die Fälle der beiden Untergruppen d) und e) gehören streng genommen nicht hierher, da die Kriegsdiensbeschädigung nicht die wesentliche Rolle hei der Entstehung der Anfälle spielte. Die Anfälle imponieren aber leicht zunächst als funktionell und geben daher gelegentlich zu diagnostischen Schwierigkeiten Veranlassung.

Bei 3 derartigen Kranken, die zur Beobachtang auf Anfälle eingewiesen waren, lag die luetische Infektion 8-10 Jahre zurück. Es hạndelte sich um Männer zwischen 28 und 35 Jahren. Abgesehen von zeitweise recht heftigen Kopfschmerzen waren bis zum Dienstantritt und in der ersten Zeit des Dienstes keine nervösen Störungen zu beobachten gewesen, vamentlich keine Anfälle. Diese traten erst nach mehr- 
monatlichem Dienst auf. Die organische Grundlage war in diesen Fällen durch leichte Störungen der Pupilleureaktion, starke Steigerung der Sehnenreflexe an den unteren Extremitäten, durch Drüsenschwollungen und positive Wassermann'sche Reaktion im Blut sehr wahrscheinlich gemacht. Aber auch die Aufälle waren von besonderer Art. Die ersten Anfälle imponierten bei diesen Kranken mit Rücksicht auf die Art der koordinierten Muskelkrämpfe und wegen der geringfügigen oder fehlenden Bewusstseinstrübung zunächst als hysterische. Der Charakter dieser Anfälle änderte sich aber bald. Die hysterischen Züge der Anfälle traten mehr und mehr zurück, die Bewusstseinsstörungen nahmen an Tiefe zu, so dass später auch lichtstarre Pupillen im Anfall beobachtet werden konnten.

In allen 3 Fällen hatte keine erbebliche Schädigung durch anstrengenden Dienst, Strapazen, Verwundungen oder Verschüttung stattgefunden; die Leute hatten vielmehr nur einige Monate Dienst gemacht, ohne besonderen Anstrengungen und Strapazen ausgesetzt gewesen zu sein. Bemerkenswert ist vielleicht noch, dass gerade solche Leute, bei denen eine beginnende organische Erkrankung des Zentrainervensystems den Anfällen zugrunde lag, ganz besondere Arbeits- und Dienstfreudigkeit zeigten und zwischen den Anfällen nur wenig allgemeine nervöse Beschwerden hatten.

In vereinzelten Fällen mussten die während der Dienstzeit aufgetretenen Anfälle von Bewusstseinsverlust mit leichten motorischen Reizerscheinungen auf eine beginnende Herzaffektion bezogen werden. In einem derartigen Falle handelte es sich um einen bereits 40 jährigen Landsturmmann, welcher vorher weder hysterische noch epileptische $\mathrm{Zu}$ stände gebabt hatte, und nun während der Dienstzeit Anfälle von Bewusstseinsverlust bekam, die dadurch besonders charakterisiert waren, dass neben der Bewusstseinsstörung ernstere Symptome von seiten des Herzens zu beobachten waren und zwar Kollapserscheinungen, kleiner fadenförmiger Puls, Zyanose der Lippen, Blässe des Gesichtes und Schweissausbruch. Die Zeichen der Herzschwäche nahmen bei solchen Anfällen gelegentlich einen bedrohlichen Charakter an. Krampferscheinungen waren dabei nicht vorhanden; es bestand nur ein leichter Tremor der Extremitäten nebst den Anzeichen einer gewissen motorischen Schwäche. Die Lichtreaktion der Pupillen war in solchen Anfällen erhalten und die spinalen Reflexe waren nicht verändert. In einem anderen analogen Falle war eine erhebliche Vergrösserung des Herzens nach links röntgenologisch zu konstatieren.

Nach Ausscheidung derartiger Fälle wit organischer Grundlage bleiben noch 189 Fälle mit funktionellen Anfällen, Krampfanfällen und 
Bewusstseinstrübungen nicht epileptischer Natur übrig. Unter diesen lassen sich zunächst die echt hysterischen Anfälle von affektiven Dämmerzuständen bei emotiven Leuten unterscheiden.

Die Hysteriker, die im lazarett bei geringfügigen An]ässen, Urlaubsentziehungen, drohender Entlassung, nach kleinen Alkoholgaben mit typischen hysterischen Anfällen reagierten, hatten sehr häufig in ibrer Vorgeschichte neuropathische oder psychopathische Züge aufzuweisen. Entweder waren schon vor der Dienstzeit vereinzelte hysterische Anfălle beobachtet worden, oder es fanden sich schwere erbliche Belastung; Kopftraumen oder nervöse Störungen schon während der ersten Entwicklung. In den Fällen, in denen bei fehlender Disposition und Belastung hysterische Anfälle aufgetreten waren, hatten auch stets während der Dienstzeit schwere Schädigungen eingewirkt und zwar: schwere Kopfverletzungen, Verschüttung, schwere seelische Erregungen, Hitzschlag und Ueberanstrengung. Als Aequivalent hysteriseher Anfälle fanden sich des öfteren Dämmerzustände, in denen von den Kranken vorangegangene selbsterlebte Kampfszenen mit entsprechenden Ausdrucksbewegungen reproduziert wurden. Derartige Zustände fanden sich besonders häufig abends zur Zeit des Einschlafens.

Die Entlassung solcher Hysteriker als zeitig dienstunbrauchbar ist wohl stets notwendig und zwar auch dann, wenn durch längere Lazarettbehandlung die typischen Aufälle für längere Zeit zum Schwinden gebracht sind. Denn die Gefahr des Rückfalles bei zu frühzeitiger Einstellung zum Dienst ist gross. Nur in den Fällen mit allerleichtesten Anfällen und leichten somnambulen Zuständen wird man die Entlassung: als arbeitsverwendungsfähig oder zu leichtem Garnisondienst verantworten können, wenn im übrigen keine erheblichen psychopathischen Zeichen bestehen und der betreffende Mann durch sein Verhalten im Lazarett gezeigt hat, dass or sich ernstlich bemüht hat, brauchbare Dienste zu leisten.

Dieser Gruppe mit echten hysterischen Anfällen stehen solche Fälle gegenüber, welche einen erheblichen Grad von Emotivität zeigen und bei denen nun als Reaktion auf äussere Ereignisse geringfügiger Art, auch nach leichten Alkoholexzessen zornmütige Erregungen auftreten, in deren Verlauf es dann nach allerhand Gewalttätigkeiten zu Bewusstseinstrübungen mit koordinierten Mnskelkrämpfen kommt. Oft hat mau in diesen Fällen den Eindruck einer willkürlichen Produktion. Beide Gruppen haben gewisse Beziehungen zueinander. Aber die Fälle mit hysterischen Anfällen zeigen meist nicht dauernd jene abnorme Affektlage, aus welcher sich bei der zweiten Gruppe die Anfälle stets entwickeln. Die Eutlassungsfähigkeit dieser Leute ist sehr schwer zu beurteilen. Sie stellen überall, im Lazarett wie bei der Truppe, eine ge- 
wisse Gefahr für die Umgebung dar. Manche derartige Leute sind durch energisches Anpacken und Androhung von Strafe doch noch im Zaume zu halten; andere reagieren darauf aber mit einer derartigen Steigerung ihrer pathologischen Reizbarkeit, dass schliesslich ibre Zurechnungsfähigkeit in Zweifel gezogen werden muss und auf Dienstunbrauchbarkeit zu erkennen ist.

Schliesslich lässt sich unter den Fällen, die zur Beobachtung auf Anfälle eingewiesen werden, noch eine Gruppe absondern, in welcher auf der Basis einer allgemeinen nervösen Erschöpfbarkeit sich Zustände von Schwindelempfindungen mit Ohnmachten und leichten motorischen Reizerscheinungen entwickeln. Die Anfangsstadien solcher Fälle sind wohl schon unter den Kranken der ersten zwei Gruppen zu finden, bei welchen, wie erwähnt, leichte allgemeine Schwindelempfindungen bei Körperanstrengungen beobachtet werden können. Dass vasomotorische Krisen in derartigen Fällen mit labilem Gefässsystem die Grundlage derartiger Zufälle abgeben können, ist von mir und anderen Beobachtern schon früher angenommen worden. Oppenheim betrachtet derartige Anfülle als Ausartung von Schwindelerscheinungen und bringt sie in Beziehung zu den sog. psychasthenischen Anfällen. Eine primäre Erkrankung des Vestibularapparates wurde natürlich zuerst ausgeschlossen. In derartigen Fälleu meiner Beobachtung fehlten stets Krampferscheinungen und andere hysterische Bewegungsstörungen. Auch die psychogene Entstehung der Anfälle war nicht zu beweisen, sondern viel häufiger gaben einfache Körperanstrengungen die Veranlassung zum Auftreten derartiger Zustände. Die Bewusstseinstrübungen waren nie vollständig: In der Anamnese dieser Leute fanden sich oft Kopftraumen und Neigung zu Alkoholexzessen; oder es waren oft schwächliche, erregbare Konstitutionen mit Neigung zu Kopfkongestionen, Herzsensationen, welche schon früher bei schweren Anstrengungen, Radfahren etc. versagt hatten und leicbt nervöse Erschöpfungssymptome gezeigt hatten.

Mehrwöcbiger Lazarettaufenthalt mit Arbeitstherapie wirkte in diesen Fällen sehr günstig, so dass oft Garnisondienstfähigkeit und Kriegsverwendungsfähigkeit erreicht werden konnten.

Gruppe 7: Funktionelle Kontrakturen im Bereiche der Extremitäten und der Rückenmuskulatur.

Von derartigen Fällen kameu nur 9 zur Beobachtung und BehandIung. Es handelte sich um Beugekontrakturen der Finger, Hand, des Unterschenkels, ferner um Kontrakturen in der Hals- und Rumpfmuskulatur, welche nach geringfügigen Verletzungen an den Extremitäten und nach Sturz auf den Rücken resp. Wirbelsäule entstanđen waren. Auch hier 
waren neben den Kontrakturen die allgemeinen Symptome der nervösen Erschöpfung und abnormen Reizbarkeit vorhanden. Die Prognose dieser Fälle war fast immer ungünstig. Die Fälle kamen allerdings erst spät in psychotherapeutische Behandlung und waren lange Zeit vorher mediko-mechanisch behandelt worden. In den meisten Fällen versagte jede Therapie. Ein hypnotischer Versuch in einem Falle mit Kontraktur des Unterschenkels führte zu einer sehr erheblichen Verschlechterung: des Zustandes und zwar insofern als durch die Hypnose ein emotioneller Schütteltremor des Beines hervorgerufen wurde, welcher längere Zeit bestehen blieb. Nur in 2 Fällen liessen sich durch Streckverband und Wachsuggestion die Kontrakturen der Finger und Hand beseitigen. Auch mit Heilbeschäftigung war oft nichts zu erreichen. 3 Fälle, in denen ich die Ueberzeugung hatte, dass die Kontraktur willkürlich produziert wurde und babituell geworden war, es handelte sich um leichte Kontrakturen in der Halsmuskulatur und der Rückenmuskulatur, habe ich zom Dienst und zwar als geeignet zum Fahrdienst entlassen.

Gruppe 8: Die funktionellen Extremitätenlähmungen.

Die funktionellen Extremitätenlähmungen nach Schussverletzungen und anderen Schädigungen stellen einen eigenartigen, häufig vorkommenden Typus einer neurologischen Affektion dar, den wir" vor dem Kriege wohl nur selten bei jungen Männern ohne hysterische Antezedentien nach Unfällen und Verletzungen zu sehen bekamen. Ob wir zur Erklärung dieser eigenartigen Lähmungen auf Grund von Kriegsbeschädigungen neue pathologische Mechanismen annehmen müssen, oder ob wir mit unseren bisherigen Vorstellungen über hysterische und neurasthenische Störungen auskommen, sol] unten eingehender erörtert werden.

Wir sehen nach leichteren Verletzungen an der Peripherie des Körpers, nach oberflächlichen Durehschüssen durch die Haut, nach Streifschüssen und Kontusionen, Verbrennungen und Ueberanstrengungen, kurz nach Verletzungen, welche weder periphere Nervenstämme noch zentrale nervöse Babnen getroffen haben können, Lähmungen eines Arms oder eines Beins oder beider Extremitäten auftreten. Ich beschränke mich hier auf Fälle mit leichten und leichtesten Verletzungen. Denn in den Fällen, in denen sich funktionelle Extremitätenlähmungen nach selır schweren Verletzungen und Zertrümmerung der Knochen und Muskeln entwickelt haben, ist es nicht leicht mit voller Sicherheit primär oder sekundär entstandene organische Beschädigungen der Nervenstämme auszuschliessen, so dass die rein funktionelle Natur der Lähmung in Zweifel gezogen werden kann. 
Ueber 7 derartige Fälle soll etwas eingehender berichtet werden.

Sehen wir uns die Symptomatologie dieser Fälle, ihre Entstehung und ihren Verlauf näher daraufhin an, welche Züge sie gemeinsam haben, und in welchen Punkten sich wesentliche Differenzen finden, so ergibt sich Folgendes: Gemeinsam ist allen Fällen, dass die Extremitätenlähmung stets eine schlaffe ist, dass die Lähmung sich stets auf der Seite der Verletzung findet, dass die elektrische Erregbarkeit für beide Stromarten ungestört ist; in allen Fällen finden sich, namentlich im Anfang, Sensibilitätsstörungen und zwar zunächst eine völlige Anästhesie und später Störungen der Schmerzempfindlichkeit; spontane Schmerzen und Druckpunkte können fehlen; eine konstante Begleiterscheinung sind angioneurotische Störungen. Die Extremitäten sind blaurot, kühl und feucht; der Handrücken erscheint oft etwas geschwollen. Die Extremitäten verlieren ihre aktive natürliche Haltung; eine geringfügige Volumabnahme in der Muskulatur ist stets zu konstatieren, es kommt aber nicht zu ausgesprochenen Atrophien einzelner Muskeln. Die Sehnenreflexe sind erhalten.

Die Symptomatologie meiner 7 Fälle ist, was das Zustandsbild angeht, eine ziemlich gleichmässige.

In der Art der Verletzung und in den uäheren Umständen, unter welchen die Lähmungen aufgetreten sind, finden sich jedoch die allergrössten Verschiedenheiten. Die Schwere und der Ort der Verletzung waren äusserst verschieden. Die Armlähmung entstand im ersten Falle nach einem Steckschuss im Akromion, im zweiten Fall nach Weichteilschuss im Unterarm, im dritten Fall nach Steckschuss in der Halsmuskulatur, im vierten Fall nach Durchschuss durch die oberen Halsmuskeln vom Gesicht aus, im fünften Falle nach leichter Kontusion und Verbrennung des linken Arms und oberflächlicher Hautverletzung an der rechten Brustseite; in diesem Falle bestand noch eine gleichseitige funktionelle Beinlähmung. Im sechsten Falle trat die Lähmung nach kurzer Anstrengung des später gelähmten linken Arms auf. Im siebenten Falle handelte es sich um eine Beinlähmung, namentlich der Extensoren des Fusses nach Weichteilschuss an der Innenseite des Oberschenkels.

Es ergibt sich also, dass der Ort und die Intensität der Verletzung für die Schwere der darauffolgenden funktionellen Lähmungen gleichgültig sind, abgeseben davon, dass die Lähmung wohl stets an der Körperseite aufzutreten pflegt, an welcher die Schädigung eingewirkt hat.

Vergleicht man nun ferner die näheren Umstände, unter welchen die Lähmungen in den verschiedenen Fällen zustande kamen. Für die 
Beurteilung der Frage, ob sie rein psychische Ursachen haben, oder ob eine mehr mechanische Erschütterung des Nervensystems als Ursache anzunehmen ist, ist die Berücksichtigung dieser näheren Umstände wichtig.

Im ersten Fall war der Mann liegend getroffen worden. Er hatte den Steckschuss am Akromion zunächst nicht bemerkt. Erst als er aufspringen wollte, machte sich die Lähmung bemerkbar. Die Schmerzen waren angeblich nicht erheblich. Der Mann war vor und nach der Verletzung nicht besonders erregt.

Im zweiten Falle befand sich der Verletzte etwas hinter der Feuerstellung bei der Feldküche, als er den Armschuss erhielt.

Im dritten Falle wurde der Mann hinter seinem Schutzschild liegend durch Schützenfeuer getrofferi.

Im vierten Falle lag der Mann im Moment der Verietzung selbst im Auschlag, als er von der Kugel getroffen wurde. Er war kurze Zeit bewusstlos; als er zu sich kam, befand er sich auf dem Truppenverbandplatz.

Im fünften Falle befand sich der Verletzte im Unterstand. Er hatte - ebenso wie die anderen eben erwähnten Fälle - bereits zahlreiche schwere Gefechte mitgemacht, war stets ruilig und kaltblütig gewesen. Seine Stellung war tagsüber nicht beschossen worden. Er stand im Unterstande, als er das Abfeuern eines Schusses konstatierte und beobachtete, wie das Geschoss herannahte. Er wurde verschüttet und erlitt leichte Kontusionen und Brandwunden an der rechten Körperseite.

Im sechsten Falle fehlte überhaupt jede psychische Erregung und jedes Ereignis, welches eine Erschütterung des Nervensystems hätte hervorrufen können. Der Manu befand sich auf dem Marsche und bemerkte beim Tragen des Gewehrs eine zunehmende Frmïdung des Arms, bis er ihm vollständig versagte. Er wurde zunächst noch einige Zeit von der Truppe mitgefülurt, im Revier bebandelt und erst nach einigen Wochen wegen der persistierenden Lähmung zurückgeschickt.

Im siebenten Falle mit einer funktionellen Lähmung des linken Beins batte ein Weichteilschuss an der Innenseite des linken Oberschenkels stattgefunden. Die Lähmung trat aber erst mehrere Monate nach der Verletzung während einer Lazarettbehandlung auf.

Es ergibt sich hieraus, dass diese Lähmungen unter den allerverschiedensten äusseren Umständen zustande kommen können, dass besonders starke psychische Erregungen im Moment der Verletzung fehlen können und dass andererseits fast in keinem meiner Fälle die Annahme gerechtfertigt ist, dass infolge einer allgemeinen Kommotio 
des Körpers und des Zentralnervensystems die Lähmung zustande kam. Auch hatten diese Verletzten nicht über besonders lebhafte Schmerzen nach der Verletzung zu berichten.

Wie steht es nun mit der Veranlagung dieser Fälle zu nervösen Störungen?

In keinem meiner Fälle war eine neuropathische oder psychopathische Disposition nachweisbar. Fs liessen sich gar keine Anhaltspunkte dafür finden, dass angeborene oder erworbene neurasthenische oder hysterische Reaktionen bereits früher bestanden hatten. Für eine latente Disposition sprach höchstens der Umstand, dass mehrere dieser Leute mit funktionellen Lähmungen eine sehr zarte und etwas weichliche Konstitution aufwiesen.

So stellt also die neuropathische Disposition keine wesentliche Vorbedingung für das Auftreten dieser Läbmungszustände dar.

Es erhebt sich nun weiter die Frage, ob sich vielleicht in der Symptomatologie dieser Fälle Anzeichen für eine rein hysterische Grundlage der ganzen Störung finden. Es kommen bier vor allem hysterische Anfälle und hysterische Sensibilitätsstörungen in Frage. Hysterische Krampfanfälle habe ich in keinem meiner Fälle beobachten können. Nur in 2 Fällen, welche ich hier nicht aufgeführt habe, da ich sie nur gelegentlich konsultativ geseben habe, fand sich neben der funktionellen Lähmung ein emotioneller Tremor der betreffenden Extremitäten, der sich gelegentlich infolge seelischer Erregung zu einem allgemeinen Schütteitremor und hysterischen Konvulsioneu steigern konnte. Was nun die Sensibilitätsstörungen angebt, so erinnerten diese tatsächlich an hysterische Anästhesien und zwar sowohl in bezug auf ihre Ausbreitung wie in der Art der Störung. Handschuhförmige oder strumpfförmige Anästhesien waren der gewöhnliche Befund. Die Intensität der Sensibilitätsstörung pflegte an den distalen Teilen der Extremitäten am stärksten zu sein und nahm nach dem Rumpf zu ab. Manschettenförmige oder inselförmige Anästhesien habe ich nicht feststellen können. Die Zone der Sensibilitätsstörung schliesst, namentlich im Anfang, die Verletzungsstelle stets in sich ein. In den Fällen, in denen der Arm selbst verletzt war, fand sich die Anästhesie am ganzen Arm, später nur an der Hand.

Im vierten Falle, in welchem sich die Verletzungsstelle an der gleichseitigen Gesichtshälfte fand, reichte die Sensibilitätsstörung bis hinauf in das Gebiet des zweiten Trigeminusastes. In diesem Falle liess sich eine scharfe Abgrenzung der Sensibilitätsstörung in der Mittellinie feststellen. In anderen Fällen waren die Angaben über die Grenzen der anästhetischen Zone sehr schwankend. 
Weitere Schlüsse auf die psychogene resp. hysterische Grundlige der Störungen ergaben sich aus dem Verlauf der Lähmungen und aus ihrer Reaktion auf psychotherapentische Beeinflussung. In drei Fällen meiner Beobachtung liess sich die Abhängigkeit der Intensität der Lähmung von rein psychischen Momenten in dem weiteren Krankheitsverlauf sicher nachweisen, während in den auderen Fällen rasche Schwankungen der Intensität der Lähmung nicht beobachtet werden konnten. Im vierten Falle war die Lähmung bereits einmal vollkommen geschwunden, so dass der Mann als kriegsverwendungsfähig entlassen war. Die Lähmung trat aber während eines Marsches zur Front wieder genau so stark auf wie vorher, so dass eine zweite längere Lazarettbehandluug notwendig war. Im fünften Falle erfuhr die Lähmung durch eine starke seelische Aufregung im Garnisondienst eine ganz erbebliche Verschlimmerung für einige Tage.

Schliesslich ist noch die Frage aufzuwerfen, wie der Eintritt solcher Lähmungen zeitlich zu den Verletzungen lag. In der Mehrzahl der Fälle wird angegeben, dass die Lähmung unmittelbar nach der Verletzung zustande gekommen sei. Diese Angaben sind natürlich in manchen Fällen nicht zuverlässig, da die Verletzung und die seelische Erregung eine genaue Selbstbeobachtung nicht immer ermöglicht und die nach der Verletzung sofort in einen Verband gelegte Extremität auf ibre Beweglichkeit nicht geprüft werden kamn. In den Fällen 1, 4, 5 und 6 wird man annebmen können, dass die Angaben der Lente, die Lähmung sei fast sofort nach der Verletzung eingetreten, zuverlässig sind; denn die gelähmte Extremität, welche in diesen vier Fällen nicht direkt oder erheblich verletzt war, wurde nicht in einen Verband gelegt, konnte also auf ihre Beweglichkeit kontrolliert werden ${ }^{1}$ ). Die Fälle 2 und 3 trugen lange Zeit Verbände, so dass man hier Zweifel haben kann, wann die Lähmung aufgetreten ist. Im 7. Falle lagen zwischen dem Eintritt der Lähmung und der Verletzung 9 Monate. Die Lähmung trat während der Lazarettbehandlung auf. Der Mann fühlte eines Morgens, dass das linke Bein, dessen grosse Fleischwunde schon längst geheilt war, eingeschlafen sei, sich tanb anfühle und willkürlich nicht bewegt werden konnte. Die Lähmung des Beins bestand dann während 6 Monaten in derselben Weise fort.

Damit ist die Symptomatologie dieser nicht komplizierten Fälle von funktioneller Extremitätenlähmung wohl in den wesentlichsten Punkten kurz wiedergegeben. Es finden sich diese funktionellen Läbmungen nun

1) Als Truppenarzt und im Feldlazarett babe ich allerdings nie derartige funktionelle Lähmungszustände zu Gesicht bekommen. - 
auch zusammen mit ausgesprochenen bysterischen und neurasthenischen Zuständen, so dass die Symptomatologie vieler derartiger Fälle sich oft mannigfaltiger gestaltet als in den mitgeteilten 7 Fällen. Auch die Fälle, in denen ausgedehnte Zertrümmerungen von. Knochen und Muskulatur vorhanden sind, und in denen neben den funktionellen Lähmungen teilweise auch organische bestehen können, bieten oft schwerere und vielgestaltigere Zustandsbilder als meine Fälle mit den leichtesten Verletzungen. Ich habe derartige Fälle hier aber, wie gesagt, nicht herangezogen, da die Verhältnisse bei ihnen viel komplizierter liegen und die organisch-bedingten Ausfallserscheinungen und funktionellbedingten oft nicht leicht auseinander zu halten sind.

Es bleibt nun schliesslich die Frage $z u$ beantworten übrig, in welcher Weise man sich das Zustandekommen solcher funktioneller Extremitätenlähmungen nach leichten Verletzungen an der Peripherie des Körpers zu denken hat. Meiner Meinung nach ist nun der Versuch verfeblt, derartige Lähmungen alle auf einen einzelnen bestimmten pathologischen Mechanismus zurückführen zu wollen. Bei dem Zustandekommen dieser schlaffen Extremitätenlähmungen kann vielmehr eine ganze Reibe sehr verschiedener ursächlicher Faktoren wirksam sein, von denen nun im einzelnen Falle bald dieser bald jener dominiert. Wir werden unterscheiden müssen zwischen den Funktionsstörungen, welche unmittelbar nach der Verletzung vorhanden sind, und solchen, welche erst später im Verlauf der Behandlung zustande kommen. Die Inaktivität der verletzten Extremitäten, die Fixierung durch den Verband während längerer Zeit müssen an sich die Trophik und Motorik der betreffenden Extremität ungünstig beeinflussen. Die Gelenke können durch das Herabhängen des verletzten und fixierten Gliedes in gewisser Weise geschädigt werden; es treten in ihnen schmerzbafte Empfindungen auf, welche den Verletzten alle Bewezungsversuche vermeiden lassen, und so zu Bewegungsstörungen nach Art der Gewohnheitslähmung führen können. Jeder weiss, dass die kurze Fixierung einer normalen Extremität in einer bestimmten Stellung schon nach kurzer Zeit unangenehme Organempfindungen macht und dass dann erneute Bewegungen Schmerzen verursachen. Ferner können leichte Atrophien in einzelnen Muskeln sehr wobl in diesen Fällen auch als arthrogene aufgefasst werden. Von sekundären neuritischen Veränderungen wird man in den oben mitgeteilten Fällen wohl kaum sprechen kömnen. In Fällen mit schweren Knochen- und Muskelverletzungen werden diese aber vielleicht eine sehr erhebliche Rolle spielen und die Prognose des Falles wesentlich verschlechtern. Die Trophik der betreffenden Extremität kann auch noch durch andere Umstände notleiden. In allen Fällen siud schwere 
vasomotorische und sekretorische Störungen, namentlich im Anfang, nachweisbar. Die gelähmten Glieder sind kübl, blaurot und feucht. Wir wissen, dass infolge länger davernder Abkühlung in der Muskulatur einer Extremität gewisse Aenderungen der elektrischen Frregbarkeit (Abkühlungsreaktion) und eine gewisse Erschwerung der Motorik (pseudomyotonische Bewegungsstörungen) schon nach kurzer Zeit zustande kommen können. Ilan hat ja bekanntlich auch von einer vasomotorischen Muskelatrophie gesprochen. Ich habe selbst früher Fälle mitgeteilt, in denen neben oder infolge funktioneller angioneurotischer Störungen leichte Abmagerang in einzelnen Muskelgebieten mit Schwächezuständen und Lagegefüblsstörungen nachweisbar waren. So können doch auch in diesen Fällen von funltionellen Lähmungen die angioneurotischen Störungen ibrerseits die Trophik und Motorik einzelner Extremitäten ungünstig beeinflussen.

Alle die eben genannten Umstände können das Zustandekommen funktioneller Schwäche- oder Lähmungszustände in einzelnen Extremitäten vermitteln; namentlich in den Fällen, in denen erst später im Verlauf der chirurgisch-orthopädischen Behandlung solche Schwächezustände konstatiert werden oder erst zur Entwicklung kommen, wird man bald diesen bald jeneu der genannten mehr sekundären Umständen eine Schuld beimessen können.

Wie erklärt sich aber das Auftreten solcher Lähmungen unmittelbar nach der Verletzung? Mehrere meiner Kranken haben mit Bestimmtheit angegeben, dass der Bewegungsausfall sofort nach der Verletzung vorhanden war.

Für diese Fälle erscheint nun die Entstehung der Lähmung auf psychogenem und emotionellem Wege am wahrscheinlichsten, da schliesslich eine gewisse seelische Aufregung im Moment des Eintritts der Verletzung in jedem Falle anzunehmen sein dürfte.

Oppenheim will nun für manche Fälle mit schweren funktionellen Lähmungen eine mehr mechanische Erschütterung des Zentralnervensystems als Ursache annehmen, welche durch überstarke Reize, sei es auf sensiblem, sei es auf sensorischem Gebiet, zustande kommt, und bleibende Funktionsstörungen in einzelnen Innervationsgebieten verursachen soll. Es soll sich also wohl um eine Störung bandeln, welche gewissermassen auf der Grenze zwischen der sogenannten Diaschisis und einer bereits organischen, molekularen Veränderung steht. Der Effekt dieser mechanischen Erschütterung soll der Verlust von Erinnerungsbildern der Bewegungsvorstellungen für die betreffende Extremität sein. Die Annahme von Oppenheim erscheint für manche Fälle mit besonders schweren Verletzungen und Verschüttungen vielleicht annehmbar. 
Für meine 7 Fälle könnte die Annahme aber nicht gemacht werden, wie sich dies aus den näheren Umständen, unter denen die Verletzungen zustande kamen, ergeben bat. Nur im Falle 5, in welchem nach schwerer Granatexplosion und Verschüttung die Lähmung entstanden war, könnte man vielleicht die Wirkung einer solchen mechanischen Erschütterung vermuten; aber dieser Fall zeigt gerade in seinem weiteren Verlauf sehr ausgesprochene hysterische Züge und liess sich durch Hypnose vollkommen heilen.

So erscheint für die Mehrzahl der Fälle doch die rein psychische Grundlage der Lähmung sehr wahrscheinlich und am plausibelsten und die Anschauung Oppenheim's von der mechanischen Erschütterung wird von den meisten Autoren mit Recht abgelehnt.

Bei der Eutstehung der Lähmungen kann aber vielleicht noch ein anderer Faktor mitwirken, auf den ich vorher schon kurz hingewiesen habe. Die meisten. Fälle zeigen starke angionenrotische Störungen, welche in manchen Fällen sich zurückbilden, in anderen Fällen aber nicht, und zwar namentlich nicht in denjenigen mit zunächst ung̈ünstiger Prognose. Aus manchen Krankengeschichten ergibt sich, dass zwischen der Intensität dieser angioneurotischen Störungen und den sie begleitenden sensiblen Störungen einerseits und der Intensität der motorischen Störungen andererseits ein gewisser Parallelismus bestebt und dass mit dem Nachlassen der schweren vasomotorischen Störungen auch die Beweglichkeit in der betreffenden Extremität eine wesentliche Besserung zeigt. Die Verletzten berichten oft, dass seit dem Moment der Verletzung die gelähmte Extremität sich eiskalt angefühlt babe und dass ihnen gleicbzeitig jedes Organgefühl für die betreffende Extremität verloren gegangen sei.

Es drängt sich nun die Frage auf, ob die angioneurotischen Störungen, die sehr wohl reflektorisch, z. B. durch Schmerzen bei der Verletzung entstanden sein könnten, diesen plötzlichen Verlust des Organgefühls in der betreffenden Extremität micht vermitteln und auf diese Weise für das Auftreten der motorischen Störungen in manchen Fällen eine Bedeutung gewinnen können.

Der naheliegende, stets gehörte Einwand ist der, dass alle diese angioneurotische Störungen sekundärer Natur sind; sie können rein psychiscbe Ursachen haben, sie können reflektorisch durch Störungen in sympathischen Zentren zustande kommen, sie können als Folge der Inaktivität der gelähmten und herabhängenden Extremität sich entwiekeln, aber jedenfalls sind sie sekundärer Natur.

Wir kennen nun aber doch eine ganze Anzahl von Angioneurosen der Extremitäten, denen eiue klinische Selbständigkeit nicht abgesprochen 
werden kann. Ich brauche kaum an die verschiedenen Formen der vasomotorischen Neurose der Extremitäten, an die Vorstadien der symmetrischen Gangrän und an die Acrocyanosis chronica anaesthetica zu erinnern. Mehr interessieren uns aber in diesem Zusammenbange die Fälle mit leichteren angioneurotischen Störungen an den Extremitäten. Bei jugendlichen Leuten beobachtet man anfallsweise auftretende Parästhesien, Kriebeln, Tanbsein verbunden mit Schwächegefühl in den betreffenden Extremitäten. Derartige Symptomenkomplexe können nach unzweckmässiger Lagerung und Fixierung einer Extremität oder nach Uebermüdung auftreten. Der Fall 6 meiner Beobachtungen, in welchem die Lähmung des Arms mit angioneurotischen Störungen nach einer kurzen Ueberanstrengung des Arms aufgetreten war, lässt sich an die erwähnte Form der vasomotorischen Neurose der Extremitäten leicht angliedern. Auch für den Fall 7 trifft dieses zu, in welchem der Kranke während einer Lazarettbehandlung morgens beim Aufwachen bemerkt, dass das linke Bein taub und gefühllos ist und nicht bewegt werden kann.

Es liegt eigentlich nahe, unsere erweiterten Erfahrungen über die vasomotorische Neurose, speziell der Extremitäten, auch auf diese Fälle von funktionellen Lähmungen anzuwenden und zu vermuten, dass diese mit grosser Regelmässigkeit vorhandenen Symptomenkomplexe mit dazu beitragen, dass in manchen Fällen solche Störungen des Organgefühls mit Paresen nach leichten Verletzungen und anderen Schädigungen zustande kommen können.

Dazu treten dann psychische Komponenten, die auf die Intensität der Störung in vielen Fällen sogar einen entscheidenden Einfluss haben, so dass die angioneurotischen Störungen gewissermassen den Verletzten nur dazu anregen, sich mit dem gestörten Organgefühl seiner Extremität zu beschäftigen und dieses weiter psychisch zu verarbeiten.

Nach alldem werden wir zur Erklärung dieser doch recht merkwürdigen Fälle funktioneller Extremitätenlähmung nach Schussverletzungen bei jungen Männern ohne alle nervöse Ȧntezedentien keine neuen patbologischen Mechanismen anzunehmen brauchen, sondern mit unseren früheren Vorstellungen über hysterische Lähmungen und angioneurotische Störungen an den Extremitäten auskommen.

Ueber die Prognose und Therapie dieser Fälle ist Folgendes zu sagen: Manche der Lähmungen sind sebr schwer therapeutisch zu beeinflussen, namentlich solche mit schweren angioneurotischen Störungen. Andere sind nach vorübergehender Besserung oder Heilung zu Rückfällen geneigt. Von therapeutischen Massnahmen kamen zur Anwendung: Beschäftigungs- und Arbeitstherapie, mediko-mechanische Behandlung, Wachsuggestion, mit Bewegungsübungen unter ärztlicher Aufsicht. 
In zwei Fällen warde leichter hypnotischer Schlaf zu Hilfe genommen, um den Kranken das Nachlassen der Bewegungsstörangen in den betreffenden Extremitäten zu suggerieren.

Wie waren nun in meinen Fällen die Heilerfolge?

Der erste Fall mit totaler Lähmung des einen Armes und starken angioneurotischen Störungen kam ungeheilt zur Entlassung. Im fünften Fall mit totaler Lähmung des rechten Armes und rechten Beines wurde nach dreimonatiger Behandlung mit Hypnose völlige Heilung erreicht. In drei Fällen war durch Lebungs- und Beschäftigungstherapie eine so. weitgehende Besserung $z u$ erreichen, dass nur noch eine leichte motorische Schwäche in den vorher total gelähmten Extremitäten nachweisbar war, und die Leute als arbeitsverwendung fähig entlassen: werden kounten. Zwei Fälle stehen noch in Rehandlung, haben sich aber soweit gebessert, dass auch bei ihnen Arbeitsfähigkeit sich wird erreichen Jassen.

Welche der genaunten therapeutischen Methoden in solchen Fällen zum Ziele führen wird, lässt sich im Voraus nicht sagen. Es ist wohl. Sache der persönlichen Erfahrung zu entscheiden, welche Methode in einem gegebenen Falle zweckmässig erscheint. Auf die Anwendung: von sehr schmerzhaften elektrischen Hautreizungen, bei deren Applikation. der Kranke festgehalten werden muss, habe ich verzichtet, auch dann, wenn der Kranke dazu evtl. seine Finwilligung gegeben haben würde. Da nach den vorliegenden Erfahrungen bei Anwendung solcher starkerelektrischer Reize Lebensgefahr nicht ausgeschlossen werden kann, so. muss eine derartige Behandlungsweise wohl prinzipiell abgelehnt werden.

\section{Gruppe 9: Die Simulanten.}

Bei der Art des Krankenmaterials meines Lazaretts war zu erwarten, dass Simulationsversuche oft vorkommen und die mala voluntas die. Prognose der meisten Fälle drïckt. Ich sehe ab von den zablreichen Fällen, welche meben tatsächlich vorhandenen nervösen Störungen auf irgend einem Gebiete die Neigung zeigten zu aggravieren und zu simulieren. Man wird selbstverständlich nicht über jeden Mann, der diese Tendenz zeigt, den Stab brechen dürfen. Die neurasthenische Erschöpfbarkeit und Erschöpfung, welche fast in allen Fällen die Basis für alle weiteren nervösen Symptome abgibt, macht solche Leute zunächst unsicher und ängstlich, wenn ihnen Anstrengungen auf körperlichem und psychischem Gebiete drohen. Da sie aber nun diese allgemeine nervöse Disposition als solche der Umgebang nicht als Krankheit demonstrieren können, so gibt ihnen das Auftreten mebr Iokalisierter Beschwerden erst die Gelegenheit und subjektiv das Recht, sich als krank zu be-- 
zeichnen; es ist begreiflich, dass sie bei der Schilderung und Hervorheben gewisser örtlicher Symptome und Beschwerden dann die Neigung haben, zu übertreiben.

Ich will vielmehr nur die Fälle hier als Simulanten aufführen, bei denen alle nerrösen Störungen als willkürlich produziert bezeichnet werden mussten. Die Zahl dieser Fälle ist nicht gross gewesen. Zwei Gruppen waren besonders häufig vertreten. Erstens Leute, welche entweder scbon vor der Einstellung geringfügige Traumen gehabt hatten und kleine Renten bezogen oder während des Kriegsdienstes ganz leichte Unfälle erlitten hatten und nun über ganz geringfügige subjektive Beschwerden klagten und behaupteten gänzlich dienstunfähig zu sein. Solche Leute melden sich häufig schon in der Ausbildungszeit krank und zeigen oft in der Mleinung, sie müssten die Dienstunbrauchbarkeitserklärung auf Grund ilıres kleinen Unfalls erzwingen können, ein sehr dreistes Benchmen. Therapeutische Bestrebungen sind in diesen Fällen nicht am Platze. Sofortige Entlassung unter energischstem Hinweis auf die Geringfügigkeit der Symptome und Unglaubwürdigkeit aller weiteren Angaben ist angezeigt. Zweitens kommt es oft vor, dass Leute mit Plattfussanlagen schwere, oft groteske Gangstörungen und Haltungs. anomalien produzieren, über Schmerzen im Fussgelenk, in den Hüften und in der Kreuzbeingegend klagen und deswegen zur Beobachtung auf Nervenleiden und Ischias eingewiesen werden. Nach genauer Untersuchung und Beratung mit dem orthopädisch-chirurgischen Sachverständigen wird man solche Fälle zum Dienst zwingen dürfen, auch wenn sie sich nicht entschliessen ihre willkürlich produzierte Gangstörung aufzugeben. Die Verwendung zum Fussdienst ist allerdings meist nicht möglich. Als Kuriosum sei angefülht, dass ein solcher Mann mit simulierter Gangstörung bei Plattfussanlage in einer leichten Angetrunkenheit seine Gangstörung vergessen hatte, in normaler Gangart abends fröhlich in das Lazarett zurückkehrte und auf diese Weise der Simulation überführt werden konnte.

leh habe damit das gesamte Krankenmaterial meines Lazaretts soweit die Neurosen in Betracht kommen - in seinen wesentlichsten Typen wiedergegeben. Die Symptomatologie der Neurosen nach Kriegsbeschädigung ist natürlich viel reichbaltiger als in diesen eben mitgeteilten leichten und leichtesten Fälien. Aber wir sehen in diesen Typen die Anfangsstadien, aus denen sich nun in anderen Fallen schwerere Krankheitszustände entwickeln können. Wir sehen auf der Basis der nervösen Erschöpfbarkeit uud Erschöpfung, deren Symptome sich schliesslich bei jedem Menschen durch körperliche und seelische 
Erschütterungen im leichten Grade und für kurze Zeiträume hervorrufen lassen, einzelne neurotische und psychotische Symptome stärker hervortreten, persistieren und in einzelnen Fällen zu erheblicher Ratwicklung gelangen. Welcher Schwellenreiz notwendig ist, um solche nervösen Reaktionen auszulösen, hängt von dem Grade der nervösen, individuell sehr verschiedenen Disposition und von der Intensität der einwirkenden Schädigung ab. Der zweite Faktor erscheint jedoch weniger wichtig, da, wie wir gesehen haben, die Art und die Schwere der Verletzung oder der Schädigung die allervariabelste Grösse ist und aus der Schwere der nervösen Störung nie ohne weiteres auf die Schwere der Schädignug geschlossen werden kaun, wie dies aus der Unfallpraxis zur Genüge bekanut ist.

Es wird ein gewisser Grad von Ansprechbarkeit des Nervensystems gegenïber den mannigfaltigen Schädigungen wohl in der Mehrzahl der Fälle angenommen werden müssen, um das Auftreten nervöser Reaktionen zu ermöglichen. Diese Disposition kann aber bis zur Einwirkung des Kriegstraumas vollkommen latent gewesen sein und braucht nur in einer sciswächlichen und wenig widerstandsfähigen psychischen und nervösen Konstitution za besteben, die vorher bei geringeren körperlichen und psychischen Ansprüchen noch keine Symptome zeitigre. Dass die Symptomatologie der Fälle bei derselben Art der Kriegsdienstbeschädigung sich einmal so, das andere Mal ganz anders gestaltet, und dass umgekebrt die verschiedensten Traumen die gleichen Symptome erzeugen können, muss auch in der Individualität des Falles selbst begründet liegen, welche wir zunächst nicht definieren können. In manchea Fällen finden sich in dem Vorleben der Kranken andeutungsweise bereits ähnliche nervöse Reaktionen, Neigung zu Anfällen, vasomotorische Krisen, lokalisierte Beschwerden von seiten des Herzens und des Magens und die durch den Dienst erzeugten nervösen Realstionen bewegen sich in denselben Bahnen. Dass in manchen Fällen der ganze Akkord nervöser und psychischer Reaktionen anklingt und in manchen Fällen nur eine Saite, muss individuelle Ursachen haben. Nur in manchen Fällen, in denen die Störungen nach besonders schweren Schädigungen aufgetreten sind, könnten vielleicht gewisse materielle Vorgänge am Nerveusystem als Ursache vermutet werden, welche nun ihrerseits ohne Vermittlung der Psyche Funktionsstörungen in einzelnen Abschnitten des Zentralnervensystems bervorrufen könnten. Denn die moderne Kriegstechnik dürfte in der Erzeugung von Schreckwirkung durch Detonation, Verschüttung oder Luftdruckwirkung alles bisher Dagewesene übertreffen. Die Vorstellung Oppenheim's, dass durch überstarke sensible Reize auf sensorischem oder sensiblem Gebiet eine zentrale Betriebsstörung Archiv f. Psyehiatrie. Bd. 57. Heft 1. 
hervorgerufen werden kann, die zwar nicht mikroskopisch erkennbar seiu dürfte, aber doch eine Verlagerung feinster Elemente bedingen kann, hat daher manches für sich. Aber, wie schon erwähnt, sind in Fällen, in denen solche schweren mechanischen Erschütterungen sicherlich nicht bestanden haben, genau dieselben schweren Symptomengruppen zu beobachten, und es ist bis jetzt nicht geglückt, Symptomenkomplexe festzustellen, welche für eine sogen. mechanische Erschütterung des Nervensystems als charakteristisch zu bezeichnen sind.

Im allgemeinen sehen wir bei diesen rein funktionellen Neurosen leichteren Grades zunächst 3 elementare Störungen primär wirksam sein: die nervöse Erschöpfbarkeit und Erschöpfung, die emotionellen oder durch affektbetonte Vorstellungen ausgelösten Störungen auf motorischem und sensiblem Gebiet und die Neigung zu depressiver Verstimmung zum Teil hypochondrischen Inhaltes. Eine von diesen Grundstörungen ist in jedem Falle nachweisbar. Die Symptome der nervösen. Erschöpfung stehen unserem Verständnis am nächsten, da sie sich aus. gewissen, noch normalen Reaktionen des normalen Seelenlebens ableiten. lassen.

Auch für die Entstehung der Zitterbewegungen und tikartigen Bewegungen der Schreckneurosen hat man eine Art Verständnis, wenn. man sie als Erimnerungskrämpfe auffasst, als eine Wiederholung von Bewegungen, die im Moment der Verletzung oder Gefahr als Abwehrbewegungen infolge eines schreckhaften Affekts auftraten. Je stärker nun im einzelnen Fall die neuropathische und psychopathische Veranlagung jst, um so mannigfaltiger gestalten sich die nervösen und psychischen Reaktionen und um so länger persistieren dieselben.

So bieten die Neurosen nach Kriegsbeschädigung, wenn man will, in bezug auf die einzelnen Symptome gar nichts prinzipiell Neues abgesehen davon, das sdie Störungen so oft vorkommen, so exzessive Grade erreichen und bei jungen Mäunern ohne hysterische und nervöse Antezedentien auftreten.

Trotz der Aehnlichkeit oder Identität der einzelnen Symptome der Kriegsneurosen mit neurasthenischen und hysterischen Symptomen wird man die Bezeichnung dieser Fälle als Kriegsneurosen doch nicht ohne weiteres zu verwerfen branchen. Die Schädigungen, welche gegenwärtig: das Nervensystem der Menschen treffen, sind qualitativ und zum mindesten quantitativ doch durchaus neu und eigenartig. Eine besondere Aetiologie pflegt immer eine gewisse Modifizierung des Verlaufs und der Gruppierung nervöser und psychischer Reaktionen zu bedingen. So ist schliesslich eine besondere Bezeichnung dieser Gruppe von Fällen, in welcher ihre Aetiologie zum Ausdruck kommt, nicht ungerechtfertigt. 
Die therapeutischen Bestrebungen müssen sich gerade bei diesen leichteren Formen von Kriegsneurosen auf das sorgfältigste dem einzelnen Falle anpassen. Dies gilt namentlich für die Auswahl und Anwendung von psycho-therapeutischen Massnahmen. Es ist Sache einer längeren persönlichen Erfahrung zu entscheiden, waun ein robustes Vorgehen unter Anwendung von Zwang und evtl. unter Betonung des Vorgesetztenverhältnisses und wann das Gegenteil angezeigt ist, wann alle therapeutischen Bestrebungen am besten eingestellt werden und eine frühzeitige Entlassung als dienstunbrauchbar am Platze ist, selbst wenu die Neigung des betreffenden Falles zu Aggravation und Simulation nachweisbar ist und zu Gegenmassnahmen und Anwendung ron $Z_{\text {wang }}$ anreizt.

Die Aufgabe eines Lazaretts, in welchem vornehmlich Arbeitstherapie getrieben wird, ist eine dreifacbe:

Erstens sollen zunächst möglichst viele Fälle als kriegsverwendungsfähig oder garnisonverwendungsfähig zur Entlassung kommen. In welchem Prozentsatz der Fälle sich dieses erreichen lässt, habe ich bereits früher mitgeteilt ${ }^{1}$ ).

Zweitens sollen die Fälle, in welchen sich eine Dienstfäbigkeit nicht mehr erreichen lässt, durch längere Behandlıng auf den böchst möglichen Grad ihrer Erwerbsfähigkeit gebracht werden, so dass diese womöglich wieder ebenso hoch ist, wie zur Zeit des Dienstantrittes und eine Entlassung ohne Rentenansprüche als arbeitsverwendungsfähig angängig ist.

Drittens hat das Lazarett die Aufgabe, ungeeignete Elemente unter den Psychopatben frübzeitig als solche zu erkennen und auszusondern, bevor solche Leute noch Ansprüche anf Dienstbeschädigung machen können, wozu sich viele dieser Elemente schon nach kurzer Dienstzeit und geringen Anstrengnngen für berechtigt halten.

Bei der Beurteilung der Dienstbeschädigungsfrage und der Abschätzung der Erwerbsbeschränkung dieser Kriegsneurosen wird es unbedingt notwendig sein, mit allen Mitteln den übertriebenen und bewusst oder unbewusst ungerechtfertigten Ansprüchen der Leute entgegenzutreten. Ueber die glatte Abweisung der simulierenden oder aggravierenden Lente braucht man kein Wort zu verlieren. Aber auch in solchen Fällen, in denen die subjektiven Beschweden noch glaubhaft erscheinen,

1) Von den in den Monaten Mai 1915 bis Januar 1916 entlassenen Leuten waren $22 \mathrm{pCt}$. kriegsverwendungsfähig, 22,6 pCt. garnisondienstfähig, $30 \mathrm{pCt}$. arbeitsverwendungsfähig und $25,3 \mathrm{pCt}$. dienstunbrauchbar. Unter den kriegsverwendungsfähig Entlassenen waren 10 pCt. Kriegsfreiwillige. 
244 Dr. Rosenfeld, Ueber Kriegsneurosen, ihre Prognose und Behandlung.

muss es gestattet sein, Entschädigungsansprüche abzulehnen, wenn bei feblendem objektivem Befund am Nervensystem die subjektiven Beschwerden nur geringfügig erscheinen oder die Umstände, welche als Dienstbeschädigungsursache angegeben werden, ihrer Natur und Schwere nach nicht geeignet erscheinen, den Gesundheitszustand eines Menschen in erheblichem Grade ungünstig beeinflussen zu können. Die geringfügigsten Umstände werden oft als Dienstbeschädigungsursache angegeben, Diätfehler, schwere Kost, anstrengender Dienst auf Kammer, leichte Aufregungen, Aerger im Dienst usw.

Ebenso wird man die psychopathische Veranlagung in vielen Fällen als ein so wesentliches Moment für die Entstehung nervöser Reaktionen bezeichnen können; dass auch aus diesem Grunde die Frage der Dienstbeschädigung oft zu verneinen sein dürfte.

Allgemejn gültige Grundsätze wird man natürlich für die Beantwortung dieser Fragen nicht formulieren können. Man wird von Fall zu Fall entscheiden müssen. Die Entscheidung sollte aber nur in der Hand von wirklich Sachverständigen liegen. Man wird nicht von jedem Arzte Fachkenntnis auf diesem Gebiete verlangen können. Die Gutachtertätigkeit in diesen Fällen von Kriegsneurosen darf nicht so gehandhabt werden, dass infolge falscher Humanität und nachgiebiger Schwäche der Energielosigkeit und hypochondrischen Aengstlichkeit der Leute Vorschub geleistet wird.

Schätzungsweise habe ich unter den 1120 Fällen meines Lazarettes 720 zusammenstellen können, in denen meiner Meinung nach in Moment der Rückkehr in den Zivilberuf eine Einschränkung der Erwerbsfähigkeit durch Dienstbeschädigung hätte abgelehnt werden müssen. Die Zahl solcher Fälle lässt sich aber sicher noch erhöhen.

\section{Literaturverzeichnis.}

Wollenberg, Nervöse Erkrankungen bei Kriegsteilnehmern. Münchener med. Wochenschr. 1914. Nr. 44.

Derselbe, Zur Lẹre von den traumatischen Neurosen. Kriegsärztliche Erfahrungen. Bruns' Beitr. z. klin. Chir: 1916. Bd. 101. H. 4.

Opponheim, Die Neurasen infolge von Kriegsverletzungen. Berlin 1916.

Horn, Ueber Schreckneurosen in klinischer und unfallrechtlicher Beziehung.

Deutsehe Zeitschr. f. Nervenheilk. 1915. Bd. 55.

Rosenfeld, Ueber einige Formen der vasomotorischen Neurose. Zentralbl. f. Neurol, u. Psych. 1901. Nr. 220; Med. Klin. 1907. Nr. 33. 